\section{Strategies for working with a preterm rabbit model of glycerol- induced intraventricular hemorrhage: strengths and limitations}

To the Editor: We read the Traudt et al. (1) article with interest, in which the authors reported their experience with our glycerol model of intraventricular hemorrhage (IVH). The authors found a much higher mortality rate and a lower incidence of IVH in the glycerol-treated E29 rabbit pups compared to our report published in 2008 (2). The higher mortality was attributed to renal toxicity and a "direct toxic action" of glycerol on multiple organ systems. We ascribe the divergence between the results of these authors and our prior observations to several differences in the experimental protocol.

Since first describing this model in 2008, we have gained experience with more than 400 litters of rabbit kits and our mortality has remained consistent at about $30 \%$ at postnatal day 14. Extremely severe IVH is one of main reasons for early mortality within $24 \mathrm{~h}$ of birth. Affected kits develop seizures and gasping respiration within a few hours of the glycerol treatment, and then expire shortly thereafter. Survivors with IVH beyond $24 \mathrm{~h}$ occasionally succumb to inadvertent passage of the feeding tube into the lung. With regard to the authors' concerns, we have performed renal function tests on glycerol-treated pups and have found them to be within normal limits apart from marginally elevated levels of blood urea nitrogen (2). We attribute this transient elevation of blood urea nitrogen to the intravascular dehydration resulting from movement of fluids from intravascular fluid to the peritoneal cavity following intraperitoneal glycerol administration.

In our opinion, the high mortality in the authors' experiments is related to their short experience in postnatal care of rabbits. The use of the correct formula, gavage feeding, minimal handling, and aseptic precautions are essential to ensure survival of these stressed animals. We have noted similar survival rates for glycerol-treated pups without IVH and matched controls untreated with glycerol (2). It is relevant to note that the authors have not shown the birth-weight or subsequent growth of the pups in this article making it difficult to comment on the possibility of dehydration, electrolyte imbalance, and poor nutrition contributing to their high incidence of death in glycerol-treated animals.
The authors also report a $46 \%$ incidence of IVH on head ultrasound as opposed to the $80 \%$ incidence reported by our laboratory (2). We agree that there is always litter-to-litter variability, just as all preterm infants do not develop IVH. IVH is less common in growth-retarded pups and in any litter with more than two to three dead pups. Moreover, small-to-moderate IVH can be missed on ultrasound evaluation. Blood in the cerebral ventricle of pups with IVH is completely cleared by day 7 and thus, small IVH can be overlooked if one were to examine pups for a ventricular bleed on necropsy after day 2. Their low incidence of IVH may also be related to lesser vulnerability of the animals to hemorrhage owing to inaccuracy of mating dates of dams or dating of the delivery. It was, surprising to find that the authors did not note seizures and death related to severe IVH, which indicates that animals were less susceptible to hemorrhage after glycerol treatment. Together, the lower incidence of IVH in the authors' experiments could be related to litter-to-litter variability, prenatal condition of pregnant rabbits, and imprecision in detection of IVH on head ultrasound.

The authors emphasize glycerol toxicity in their paper. They noted very high levels of glycerol in the liver and forebrain shortly after treatment, but they did not look for the evidence of glycerol-induced injury in these organs. Conversely, we have reported a lack of evidence of inflammation, cell-death, or neuronal degeneration in the forebrain of glycerol-treated pups without IVH as opposed to pups with glycerol-induced IVH (2). Glycerol is widely used in pharmaceutical formulations and also as a therapeutic agent. Intravenous glycerol therapy ( $25 \mathrm{~g} /$ dose) has been used to treat ischemic brain edema in adult humans which elevates the cerebral glycerol level to $350 \%$ without influencing cerebral glutamate, lactate, or pyruvate (3). Oral glycerol $(6 \mathrm{~g} / \mathrm{kg} / \mathrm{d})$ has been used as an adjuvant therapy in children with meningitis (4). Had glycerol been as toxic as purported, it would not have been used to treat brain tumors, trauma and infection, or as a peritoneal dialysate in humans $(5,6)$. The authors also noted $13 \%$ and $27 \%$ elevation in total glutathione in the cerebral cortex at 1 and $6 \mathrm{~h}$ of age, which may be statistically insignificant. Our Chua et al. (7) paper has been misquoted in the discussion section by the authors to point out that glycerol disrupts oligodendrocyte maturation. Conversely, we have reported that maturation of oligodendrocytes is suppressed in pups with glycerol-induced IVH compared with glycerol-treated controls without IVH (8).

Traudt and colleagues have concluded that glycerol decreases the total brain and cerebellar volume at day 12 in a dose-dependent manner, independent of the presence of IVH. The number of P12 survivors were 7, 3, and 5 in $3.25,4.87$, and $6.5 \mathrm{~g} / \mathrm{kg}$ glycerol dose groups, respectively (their Table 1) and of these, three pups with IVH were excluded from evaluation (1). In view of the small sample size of each group, unknown birthweight and growth of animals, and a presumably sick group of 


\section{Correspondence | Ballabh and LaGamma}

pups exhibiting high mortality, the authors' conclusion might be premature. Authors have shown reduction in brain and cerebellar volume after glycerol treatment; however, a doseresponse relationship of glycerol and brain volume is unclear.

In closing, despite glycerol causing certain molecular and morphologic changes in brain, this model of glycerol-induced IVH is extremely valuable; and we have employed this model in making important discoveries in the field of IVH. We have found consistent hypomyelination in all pups with moderateto-severe IVH at day 14, while myelination is preserved in glycerol treated controls without IVH. More importantly, we have performed pharmacologic intervention in pups with moderate-to-severe IVH and have found that suppressing inflammation by COX2 inhibition, inhibiting bone morphogenetic protein by human recombinant noggin, or treatment of thyroid hormone restores white matter injury $(8-10)$. This information can be translated into clinical trials and enhance the quality of life of infants with IVH. Should the authors have questions, they may contact us by e-mail or phone.

Disclosure: There is no conflict of interest to disclose.

\section{Praveen Ballabh ${ }^{1}$ and Edmund F. LaGamma ${ }^{2}$}

${ }^{1}$ Department of Pediatrics, Cell Biology and Anatomy, New York Medical College-Maria Fareri Children's Hospital at Westchester Medical Center, Valhalla, New York; ${ }^{2}$ Department of Pediatrics, Biochemistry and Molecular Biology, New York Medical College-Maria Fareri Children's Hospital at Westchester Medical Center, Valhalla, New York.
Correspondence: Praveen Ballabh (pballabh@msn.com)

\section{REFERENCES}

1. Traudt CM, McPherson RJ, Studholme C, Millen KJ, Juul SE. Systemic glycerol decreases neonatal rabbit brain and cerebellar growth independent of intraventricular hemorrhage. Pediatr Res 2014;75: 389-94.

2. Georgiadis $\mathrm{P}, \mathrm{Xu} \mathrm{H}$, Chua $\mathrm{C}$, et al. Characterization of acute brain injuries and neurobehavioral profiles in a rabbit model of germinal matrix hemorrhage. Stroke 2008;39:3378-88.

3. Berger C, Sakowitz OW, Kiening KL, Schwab S. Neurochemical monitoring of glycerol therapy in patients with ischemic brain edema. Stroke 2005;36:e4-6.

4. Molyneux EM, Kawaza K, Phiri A, et al. Glycerol and acetaminophen as adjuvant therapy did not affect the outcome of bacterial meningitis in Malawian children. Pediatr Infect Dis J 2014;33:214-6.

5. Smit W, de Waart DR, Struijk DG, Krediet RT. Peritoneal transport characteristics with glycerol-based dialysate in peritoneal dialysis. Perit Dial Int 2000;20:557-65.

6. Unterberg AW, Stover J, Kress B, Kiening KL. Edema and brain trauma. Neuroscience 2004;129:1021-9.

7. Chua CO, Chahboune $\mathrm{H}$, Braun A, et al. Consequences of intraventricular hemorrhage in a rabbit pup model. Stroke 2009;40:3369-77.

8. Dummula K, Vinukonda G, Chu P, et al. Bone morphogenetic protein inhibition promotes neurological recovery after intraventricular hemorrhage. J Neurosci 2011;31:12068-82.

9. Vinukonda G, Csiszar A, Hu F, et al. Neuroprotection in a rabbit model of intraventricular haemorrhage by cyclooxygenase-2, prostanoid receptor-1 or tumour necrosis factor-alpha inhibition. Brain 2010;133(Pt 8): 2264-80.

10. Vose LR, Vinukonda G, Jo S, et al. Treatment with thyroxine restores myelination and clinical recovery after intraventricular hemorrhage. J Neurosci 2013;33:17232-46. 